\title{
Elastic and inelastic neutron scattering for the study of magnetic thin films and heterostructures
}

\author{
Karine Dumesnil \\ Institut Jean Lamour (UMR CNRS 7198), Université de Lorraine, Vandoeuvre les Nancy, France
}

\begin{abstract}
Neutron-based techniques have proved for decades to be essential tools in the investigation of magnetic materials. The strong experience gathered in neutron centers together with high performance instruments have also largely contributed to the researches on magnetic nanosystems. We will present here a few examples of the use of elastic and inelastic neutron scattering for the study of Dysprosium-based thin films and heterostructures. Elastic neutron scattering in rare earth-based nanosystems provides crucial information both on the crystal structure and the modulated magnetic order. This has especially permitted to connect the drastic change in Curie temperature observed in Dy epitaxial films to modifications of both magnetoelastic and exchange contributions. In superlattices, elastic neutron scattering has been successfully used to analyze the magnetic coupling phenomena, in particular the long range coherent propagation of the Dy helical order. The example of Dy/Er superlattices highlights this fascinating coupling mechanism through either a paramagnetic or magnetic spacer. Inelastic Neutron Scattering is the technique of choice to investigate magnetic excitations over the entire Brillouin Zone. The very low inelastic scattering cross section can however make any investigation of nanosystems very difficult and very few studies have indeed been reported so far on nanosystems. We will show that relevant information could be obtained from high quality single crystalline Dy films and superlattices. These promising results are paving the way to new spin wave explorations in a wider range of magnetic nanosystems.
\end{abstract}

\section{INTRODUCTION}

Research on magnetic nanosystems has considerably expanded those last decades, due both to the rapid development of nanotechnology, enabling the elaboration and better control of always more sophisticated low dimension systems [1], and to their interest for practical applications, mainly in the domain of data storage and data processing [2].

The vast majority of magnetic nanosystems are based on magnetic $3 \mathrm{~d}$ transition metals (TM) and theirs alloys, one advantage being of course their high Curie temperature, a key point for the development of nanodevices for applications. But in order to extend their potential functionalities, magnetic nanosystems now generally combine different types of materials (metallic, oxydes, ...) with specific properties (magnetic, ferroelectric, semiconductor, superconductor ...) that may be coupled and give rise to new and exciting phenomena.

In this general context, pure rare earth (RE) metals can hardly compete in the domain of high Curie temperature and high polarization, mainly because their magnetism arises from $4 \mathrm{f}$ strongly 


\section{Collection SFN}

localized moments that can only indirectly interact via polarization of conduction electrons, the so-called RKKY coupling. Those specificities make however rare earth metals of particular interest in magnetic nanosystems: the strong interaction between the $4 \mathrm{f}$ cloud and the crystal lattice are at the origin of strong magnetocrystalline anisotropy and magnetoelastic effects that occur in pure rare earth metals but also in rare earth based alloys. Some layered RE/TM systems and RETM thin films are thus known to exhibit strong perpendicular anisotropy; $\mathrm{REFe}_{2}$ compounds present extremely large room-temperature magnetostriction [3] and could be interesting candidates in the development of nano-actuators and of so-called artificial multiferroic in which lattice strains are monitored by coupling to a piezoelectric material. The antiferromagnetic coupling between heavy RE and TM net magnetization also enables the engineering of a magnetic compensation point where the magnetization drops to zero, a property one can interestingly take advantage of in spintronic and spin torque devices [4]. Some Sm-based intermetallics even exhibit intrinsic magnetic compensation that amazingly coexists with long range ferromagnetic order [5-7].

In magnetic nanosystems, rare earth metals also raise particular interest due to their large magnetization and non-collinear magnetic order. As prototypes of spin-spiral magnetic phases, heavy RE have been pointed out as potential materials for testing novel magnetoelectric phenomena predicted in non-collinear magnetic systems [8,9]. Their intrinsically inhomogeneous magnetic structures may constitute model systems to explore for instance the effects arising when a spin-polarized current travels through a material with non-collinear magnetization [10]. Moreover, helical domains in RE metals have been recently predicted to give rise to nontrivial domain walls consisting of a regular array of vortex lines, exciting objects that could be driven by electric current [11]. Helical domains also constitute model systems to investigate dynamics and magnetic rearrangement that may occur in ordered antiferromagnets nanosystems [12].

The goal of this presentation is to provide examples of interesting phenomena occuring in rareearth based magnetic nanosystems. We will focus on epitaxially grown dysprosium-based nanosystems [13], chosen to explore the effect of epitaxial strains, reduced dimension and magnetic coupling on magnetic properties. We will also essentially focus on the results that we could obtain from neutronbased investigations.

Simultaneously to nano-fabrication processes, the techniques to investigate magnetic nano-objects have largely evolved to enable the investigation of small and complex hybrid systems. Synchrotronbased techniques have especially raised considerable interest, mainly because of the achievable high brilliance and resolution, and of the tunable incident photon energy that allows chemical and orbital selectivity. In terms of magnetic scattering, elastic neutron scattering remains however particularly interesting since the large magnetic signal can equal the nuclear one. Elastic neutron scattering in RE-based nanosystems gives essential information both on the crystal structure and the helical order, as it will be shown for Dy epitaxial films (Sect. 2). It permits to analyze the magnetic coupling in superlattices, especially the potential coherent propagation of the Dy helical order through a magnetic or non-magnetic spacer (Sect. 3). Inelastic Neutron Scattering (INS) is the technique of choice to investigate magnetic excitations over the entire Brillouin Zone, but suffers from a very low inelastic scattering cross section that can make any investigation of nanosystems very difficult. The studies of magnetic excitations in nanosystems are thus still rare and limited $[14,15]$. We will show in this example that relevant information have been recently obtained in high quality single crystalline Dy thin films and superlattices (Sect. 4).

\section{EPITAXIAL STRAINS AS A WAY TO TUNE MAGNETIC ORDER IN DYSPROSIUM FILMS}

Bulk Dy orders from the paramagnetic to the helical state at $T_{N}=179 \mathrm{~K}$. Below this temperature, the magnetic moments are in the basal plane and the helix wave vector is along the c-axis. The turn angle 

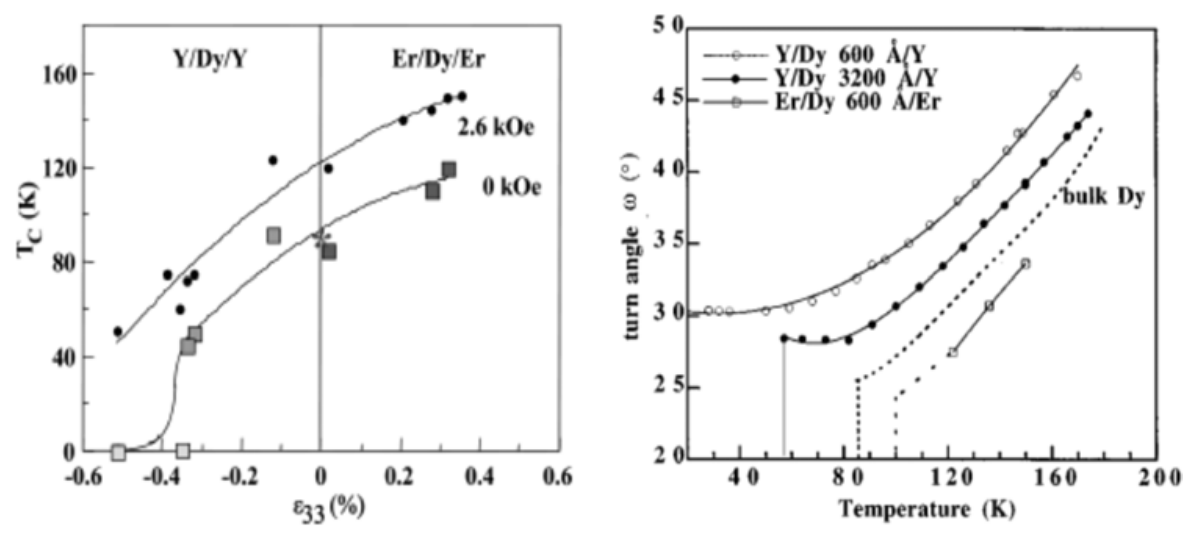

Figure 1. Left: variation of $T_{c}$ for Dy films grown between $Y$ or Er as a function of c-axis epitaxial strain. Lines are guide to the eyes. Right: temperature dependence of turn angle between Dy moments in Y/Dy/Y and Er/Dy/Er trilayers. The dotted curve represents variation in bulk Dy.

decreases from $44^{\circ}$ just below $\mathrm{T}_{\mathrm{N}}$ to $26^{\circ}$ at $\mathrm{T}_{\mathrm{C}}=89 \mathrm{~K}$ where it drops to $0^{\circ}[16,17]$. The modulated helical order is known to originate from a specific nesting of the Fermi surface that makes the generalized susceptibility of conduction electrons maximum for a given wave vector $\mathrm{Q}_{\text {mag }}$ parallel to the c-axis. The exchange energy is the smallest for a wave vector equal to this non-zero $\mathrm{Q}_{\text {mag }}$ value yielding the stabilization of a modulated magnetic order. The difference in exchange energy between the modulated and the collinear phases is related to the turn angle observed in the helical phase. The ferromagnetic transition at $89 \mathrm{~K}$ is driven by magnetoelastic effects [18] that balance the exchange interaction provided by the conduction electrons still favoring the modulated phase. The transition is accompanied by an orthorhombic distortion along the in-plane easy magnetization axis and a large expansion along c [19].

The goal of this study was to investigate how epitaxial strains would affect the magnetism in Dy films. Since the various magnetic phases in Dy result from a delicate balance between exchange, magneto-elastic and magneto-crystalline energy terms, epitaxial strains were supposed to play a determining role in magnetic order. The idea was to explore a range of films with epitaxial strains going from negative to positive values. For this purpose, advantage was taken from the very close crystal structures of different heavy rare earths and close metal like Yttrium. Dy epitaxial films, ranging in thickness from $10 \mathrm{~nm}$ to $350 \mathrm{~nm}$, have been sandwiched either between Y layers (50 nm thick) or between Er layers (100 nm thick). Both Y and Er exhibit hcp crystal structure, as bulk Dy, but their lattice constants are respectively $+1.56 \%$ larger and $-0.9 \%$ smaller than bulk Dy ones. The Dy films are thus submitted to epitaxial strains of opposite signs, as evidenced from X-ray scattering: In $\mathrm{Y} / \mathrm{Dy} / \mathrm{Y}$ (Er/Dy/Er), Dy is expanded (compressed) in the plane, which results in a c-axis compression (expansion) along the growth direction. The magnitude of the strains of course depends on the Dy thickness, as expected from its coherent growth. Neutron scattering experiments (G4.3, LLB, France) with the scattering vector $\mathbf{Q}$ parallel to the [0001] growth direction have been performed to understand how Dy magnetic properties are affected by those expitaxial strains and how these modifications can be related to magnetoelastic and exchange energy contributions [20,21]. Magnetic scattering from a c-axis modulated magnetic phase yields the appearance of magnetic satellites around nuclear Bragg peaks along magnetic propagation direction, i.e. [0001] in the present case. The positions of these satellites respect to the nuclear Bragg peak are directly related to the magnetic wave vector, i.e. to the turn angle.

Figure 1 gathers the most demonstrative results, i.e. the variation of the Curie temperature versus the epitaxial strain along the growth direction $\left(e_{33}\right)$ and the temperature dependence of the turn angle for three representative systems. 


\section{Collection SFN}

It appears that the helical phase is stabilized in $\mathrm{Y} / \mathrm{Dy} / \mathrm{Y}$ (decrease of $\mathrm{T}_{\mathrm{c}}$ ) while its range of existence is reduced (increase of $T_{c}$ ) in Er/Dy/Er. The ferromagnetic phase is even totally supressed for negative strain larger than $-0.3 \%$, i.e. for the thinnest films grown between Y layers. The turn angle in Dy films decreases with temperature, as in bulk Dy, because magnetic ordering causes opening of super-zone gaps in Fermi surface. But most interesting are the shifts of the turn angle compared to bulk values, in opposite ways for the two systems: larger than bulk for $\mathrm{Y} / \mathrm{Dy} / \mathrm{Y}$ and smaller than bulk in Er/Dy/Er. This shift is observed even at high temperature where magnetoelastic effects are negligible, which suggests an influence of epitaxial strains on the Fermi surface and consequently on the exchange energy barrier between modulated and collinear phases.

Those modifications of the magnetic order in epitaxial Dy films have been unambiguously related to the role of epitaxial strains and clamping of the film crystal lattice to the substrate [20]. In both systems, lattice clamping tends to limit the in-plane orthorhombic distortion and thus to delay the ferromagnetic transition, as observed in a different scattering geometry with scattering vector in the growth plane. Magnetoelastic effects have been investigated through the variation of the c lattice parameter as a function of temperature. It appears that epitaxial strains contribute to modifications of both magnetoelastic and exchange energies; however they have opposite effects in the two systems: In Er/Dy/Er trilayers, epitaxy tends to strain the Dy lattice in a similar way compared to bulk ferromagnetic Dy and thus favors the ferromagnetic order from magnetoelastic point of view, despite the limited orthorhombic in-plane distortion. The exchange energy barrier is lowered, because of changes in the Fermi surface induced by epitaxial strains. In Y/Dy/Y, all effects are in favor of the modulated phase (decreasing $\mathrm{T}_{\mathrm{c}}$ ): the sign of epitaxial strains are opposite to those observed in bulk, the orthorhombic distortion is limited by clamping and the exchange energy barrier is larger than in bulk.

A model based on the different energy contributions has been developed to account for the shift of $\mathrm{T}_{\mathrm{c}}$ observed when Dy is epitaxially grown either on $\mathrm{Y}$ or $\operatorname{Er}[21]$.

\section{MAGNETIC COUPLING IN DY-BASED SUPERLATTICES}

In layered systems composed of one or several magnetic materials arises the question of the interactions between successive magnetic layers. In the case of ferromagnetic layers separated by non-magnetic ones, magnetostatic interactions can lead to either antiparallel or parallel arrangements, this latter case originating from "orange-peel" coupling via interfaces roughness. Long range coupling might also establish through a non-magnetic layer via a RKKY mechanism that exhibits an oscillatory behavior as a function of the spacer thickness. The magnetic arrangement between consecutive ferromagnetic layers then turns out to be either parallel or antiparallel, depending on the thickness of the non-magnetic spacer.

A similar question arose in RE-based superlattices where magnetic layers exhibit more exotic non collinear magnetic orders. How do those specific phases interact from one layer to the other and can any long range order establish throughout the system? Elastic neutron scattering was extensively used to address those issues. The signature of long range modulated order in such periodic systems is indeed rather straightforward: when the modulated order is confined to individual Dy layers, broad magnetic satellites are observed; when a long range modulated order develops, the magnetic coherence length increases and broad satellites are replaced by a set of much narrower magnetic satellites whose relative positions are related to the SL periodicity, i.e. the bilayer thickness [22].

As far as Dy-based systems are concerned, the first superlattices to be explored combined Dy with a non-magnetic element: Dy/Y [23] and Dy/Lu [24]. As mentioned previously, Y crystal lattice is larger than Dy and thus tends to expand the Dy lattice in the (0001) plane. Lu crystal lattice is smaller than Dy one, as in the case of Er, and thus tends to compress the Dy lattice in the (0001) plane. Consistently with the influence of epitaxial strains reported in Section 2, the Dy helical phase is favored (disfavored) in Dy/Y (Dy/Lu) superlattices: it extends towards lowest temperature in Dy/Y superlattices 


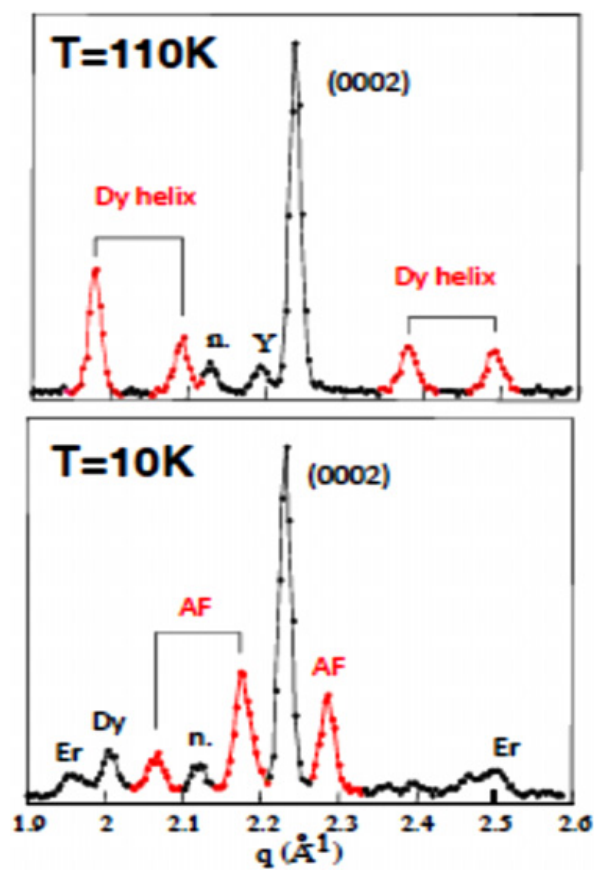

Figure 2. Elastic neutron scattering spectra collected at $110 \mathrm{~K}$ and $10 \mathrm{~K}$ for a $\operatorname{Dy}(3.5 \mathrm{~nm}) / \operatorname{Er}(2.3 \mathrm{~nm})_{50}$ superlattice.

and only in a narrow temperature range below $\mathrm{T}_{\mathrm{N}}$ in Dy/Lu superlattices. But the main result was the observation of the coherent propagation of the Dy helical phase through non-magnetic Y or Lu: the portions of magnetic helix in successive Dy layers are not independent of each other but do exhibit a phase relation, as if a virtual helix establishes in the non-magnetic layers. It has been shown that this virtual helix corresponds to the stabilization of a spin-density wave in the conduction band of Y (Lu); the associated turn angle (wave vector) in non-magnetic layers is thus different from the one in Dy and is related to specific features of $\mathrm{Y}(\mathrm{Lu})$ Fermi Surface. The long range coherence of Dy helical order is observed as far as the spacer thickness does not exceed approx. $10 \mathrm{~nm}$ and generally extends over several bilayers.

Beyond the investigation of Dy/non-magnetic systems, the study of Dy/Er superlattices comprising two magnetic RE was undertaken [13]. At the difference of Dy, bulk Er exhibits an axial anisotropy along the c-axis. It orders at $85 \mathrm{~K}$ in a c-axis modulated phase, develops an in-plane component below $52 \mathrm{~K}$ and presents a transition to a conical phase with c-axis ferromagnetic component at $20 \mathrm{~K}$. In this specific system with competing anisotropies, the issue of coherent propagation of Dy in-plane modulated order could be addressed both through paramagnetic and magnetic Er [13, 25, 26].

Figure 2 presents two elastic neutron scattering spectra that have been measured at $110 \mathrm{~K}$ and $10 \mathrm{~K}$ with the scattering vector along the c-direction for a $[\mathrm{Dy}(3.5 \mathrm{~nm}) / \operatorname{Er}(2.3 \mathrm{~nm})]_{50}$ superlattice. At $110 \mathrm{~K}$, two sets of peaks (Dy helix) of magnetic origin appear on either side of the nuclear main (0002) Bragg peak. The occurrence of magnetic satellites reveals the presence of the helical ordering in the dysprosium layers. The fact that they are split into several peaks separated by $2 \pi / \Lambda$ proves that the helix propagates coherently through paramagnetic erbium layers ( $\Lambda$ is the chemical periodicity). The fit of the spectra using magnetic scattering factors involving both the wave vector of the helical phase in Dy layers and the wave vector of the spin density wave in Er layers shows that the effective turn angle in Er layers is 


\section{Collection SFN}

independent of temperature and is approximately equal to the maximum of the generalized susceptibility of the conduction electrons in bulk Er.

This evidences the stabilization of a spin-density wave in the conduction band of paramagnetic Er, which then couples to the local moments on the dysprosium sites. From the width of these magnetic peaks, one can deduce that the magnetic coherence length is approx. $55 \mathrm{~nm}$ at $110 \mathrm{~K}$, i.e. more than 9 bilayers thickness. On the other hand, as already observed in other systems, the magnetic coherence length of the helical phase of dysprosium is a function of the thickness of the spacer. For an erbium spacer thickness of $11.1 \mathrm{~nm}$, the width of the magnetic satellites encompasses the bilayers harmonic position rendering them unresolved: the basal plane magnetic coherence at $110 \mathrm{~K}$ is limited to one dysprosium layer.

At $10 \mathrm{~K}$ new peaks (referred to as AF in Fig. 2) are observed at positions corresponding to a modulation twice of the chemical modulation. These peaks are the sign of an antiparallel arrangement between dysprosium ferromagnetic layers. The coherence length of this AF phase is $30 \mathrm{~nm}$ at $10 \mathrm{~K}$. Such antiparallel coupling between the magnetic layers has been previously observed in the Dy/Lu system and explained as the result of dipolar interactions associated with the formation of orthorhombic distorted domains. Whether this interpretation is still valid in the present case where Er presents an ordered complex magnetic phase and where orthorhombic distortion appears to be blocked is questionable. For smaller Er spacer thickness $(1.1 \mathrm{~nm})$, the low temperature arrangement between ferromagnetic Dy layers appears to be parallel, which has been also observed in Dy/Lu superlattices with thin Ho spacer layer.

\section{MAGNETIC EXCITATIONS IN DY-BASED NANOSYSTEMS}

The study of magnetic excited states allows a deeper understanding of magnetic materials at a microscopic level, especially the determination of key parameters such as exchange coupling, anisotropy or magneto-elastic constants which control the dynamical properties. This is of fundamental importance, especially in the context of applications where the capability to design new functional materials depends on this microscopic understanding. Inelastic Neutron Scattering has proven to be an indispensable tool for the study of magnetic excitations [27-30], the only known that is able to measure excitations across the entire Brillouin zone, and can give a comprehensive explanation of why a material has its magnetic properties.

However, the INS cross section is far smaller than the elastic one and up to now, very few INS experiments have been performed on reduced dimension systems [14, 15]. The study of magnetic excitations in magnetic nanosystems has been mainly limited to ferromagnetic resonance and Brillouin light scattering experiments that can only probe spin waves at or close to the Brillouin zone center, and thus do not enable the determination of dispersion of spin waves in the whole BZ. The exploration of spin waves with much smaller wavelength is possible with INS. Those spin waves with wavelength in the range of a superlattice periodicity can be dominated by exchange coupling between magnetic layers and INS may thus open up the possibility to study the dynamic behavior of the exchange coupling in magnetic superlattices [31]. With measuring magnons over a wide range of wavelengths, INS applied to magnetic nanosystems is a unique tool to investigate magnetic interactions both within and between magnetic layers and to explore whether magnetic fluctuation waves propagate throughout the system.

The main goal of the current study was to investigate the magnetic excitations in Dysprosium-based nanosystems, especially to analyze how reduced dimensions, strains and magnetic coupling may affect spin waves and how this can be related to changes in intrinsic properties. Dy-based nanosystems have been chosen because both the large magnetic moment of Dy and the high crystal quality of MBE grown single crystalline systems may help overcoming the low inelastic cross section. Moreover, specific samples have been fabricated over a large substrate area so that four $4 \mathrm{~cm}^{2}$ pieces have been cut and 
precisely co-aligned in a dedicated sample holder, thus increasing the amount of available material. A $3 \mu \mathrm{m}$ thick Dy epitaxial film has been first measured as a test sample. The effects of reduced dimension and magnetic coupling have then been explored in Dy/Lu superlattices with $5 \mathrm{~nm}$ thick Dy layers. Varying the thickness of individual $\mathrm{Lu}$ spacer layers $(18 \mathrm{~nm}$ and $3 \mathrm{~nm})$ permits us to build stackings of either non-interacting or coupled thin Dy layers. Experiments have been performed on the 4F2 triple axis spectrometer at LLB, mainly with $\mathrm{kf}=1.55 \AA^{-1}$.

\subsection{Thick dysprosium epitaxial film}

NS spectra have been recorded between $110 \mathrm{~K}$ and $30 \mathrm{~K}$ and in two different geometries: with the scattering vector along the perpendicular to the film $\mathbf{c}^{*}$ direction and parallel to the in-plane $\mathbf{a}^{*}$ direction. A set of representative measurements are given in figure 3 for $\mathrm{T}=100 \mathrm{~K}$ (helical phase) and $\mathrm{T}=30 \mathrm{~K}$ (ferromagnetic phase). They have been measured in collecting successive energy scans for different $\mathrm{Q}$ values. Experimental results are compared to a calculation from diagonalization of the Spin Hamiltonian in the harmonic approximation. This calculation considers a ferromagnetic coupling in hexagonal basal planes and between first neighbors along the c-axis. An antiferromagnetic coupling is used between second neighbors along c. It takes account of the easy-plane anisotropy and of supplemental in-plane axial anisotropy in the ferromagnetic phase.

Experiments reveal clear dispersion curves that are in good agreement with the qualitative shapes expected from calculation and with results reported along $\mathbf{c}^{*}$ for bulk dysprosium [32]. Note that, to the best of our knowledge, those are the first results ever reported along $\mathbf{a}^{*}$ for Dy.

In the helical phase $(100 \mathrm{~K})$, two dispersion curves are clearly observed when the scattering vector is parallel to the magnetic propagation vector (along $\mathbf{c}^{*}$ ). Their origins are the satellites observed at $\pm \mathrm{q}_{m}$ from (0002) nuclear peak in an elastic scattering experiment. Because the ions in the two sublattices of hcp lattice form equally separated planes along this direction, those correspond to the superimposition of single acoustic branches [33]. Along $\mathbf{a}^{*}$, multiple branches are observed, in agreement with calculation. Dispersion curves do not drop to zero, consistently with the absence of elastic magnetic satellites in this direction, perpendicular to the magnetic propagation direction. In the ferromagnetic phase $(30 \mathrm{~K})$, the dispersion curves are drastically different and two main features can be noticed: (i) There is a clear gap opening at (002) and (101) whose magnitude is related to the strong coupling to the crystal lattice [34], i.e. magnetocrystalline and magnetoelastic constants. The gap value is very similar to the one reported for bulk Dy and exhibits the same increase with decreasing temperature (approx. $3.3 \mathrm{meV}$ at low temperature), as expected from increased anisotropy. (ii) The dispersion curve along c* reveals local minima off the zero magnon wave vector in contrast to what could be expected from a collinear phase. Since the shape of dispersion curves are directly related to Fourier Transform of indirect exchange interaction [32], these local minima reveal that exchange interaction is maximum for a wave vector different from zero which in fact corresponds to the magnetic wave vector in the helical phase. The ferromagnetic phase therefore establishes despite exchange interaction that would still favor a modulated phase. This is in perfect agreement with the well-known picture of ferromagnetic transition in dysprosium: it is driven by magnetoelastic contributions while indirect exchange mediated by conduction electrons is at the origin of the helical phase.

\subsection{Reduced dimensions and magnetic coupling in Dy/Lu superlattices}

The study of magnetic excitations has been then extended to two Dy/Lu superlattices: $[\operatorname{Dy}(5 \mathrm{~nm}) / \mathrm{Lu}(18 \mathrm{~nm})]_{193}$ and $[\mathrm{Dy}(5 \mathrm{~nm}) / \mathrm{Lu}(3 \mathrm{~nm})]_{597}$. Elastic neutron scattering $(6 \mathrm{~T} 2$, LLB) have first confirmed that below Curie temperature, ferromagnetic Dy layers were decoupled from each other for thick Lu spacer and present long range antiparallel coupling for thin Lu spacer. 


\section{Collection SFN}
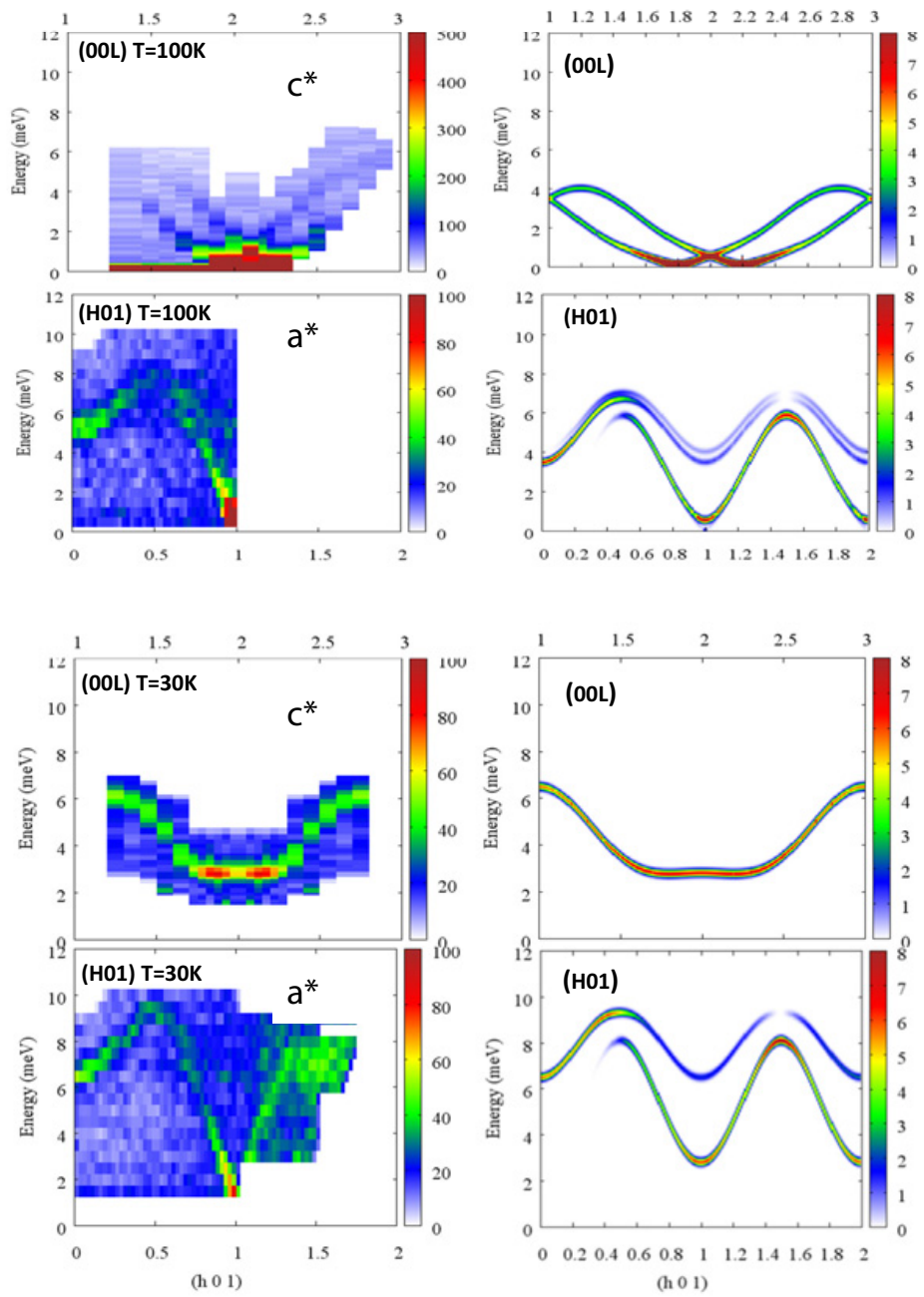

Figure 3. Dispersion curves at $100 \mathrm{~K}$ (top two rows) and $30 \mathrm{~K}$ (bottom two rows) for a $3 \mu \mathrm{m}$ thick Dy film. Left column correspond to experimental results, right column correspond to calculation (see text). First and third rows correspond to scattering vector along (00L), i.e. parallel to $\mathbf{c}^{*}$. Second and fourth rows correspond to scattering vector along (H01), i.e. parallel to $\mathbf{a}^{*}$.

INS measurements have been performed with the scattering vector along $\mathbf{c}^{*}$ and exclusively in the temperature range where individual Dy layers order ferromagnetically, i.e. below $157 \mathrm{~K}$ in $[\mathrm{Dy}(5 \mathrm{~nm}) / \mathrm{Lu}(3 \mathrm{~nm})]_{597}$ and below $176 \mathrm{~K}$ in $[\mathrm{Dy}(5 \mathrm{~nm}) / \mathrm{Lu}(18 \mathrm{~nm})]_{193}$. The enhanced Curie temperature compared to bulk Dy $(89 \mathrm{~K})$ is related to its strain state in Dy/Lu systems, the Dy crystal lattice being all the more strained than Lu spacer is thick.

The first important result is that we could actually measure dispersion curves from these nanosystems, i.e. from stackings of only $5 \mathrm{~nm}$ thick Dy films. The signal was more difficult to extract in the $18 \mathrm{~nm}$ thick Lu superlattice because of the smaller number of bilayer repeats. The dispersion curves measured for ferromagnetic Dy spin waves in SL's have been reported in Figure 4 with the results from the thick Dy film added for comparison. Dispersion curves are obtained from a fit of energy scans performed for different $\mathrm{Q}$. 

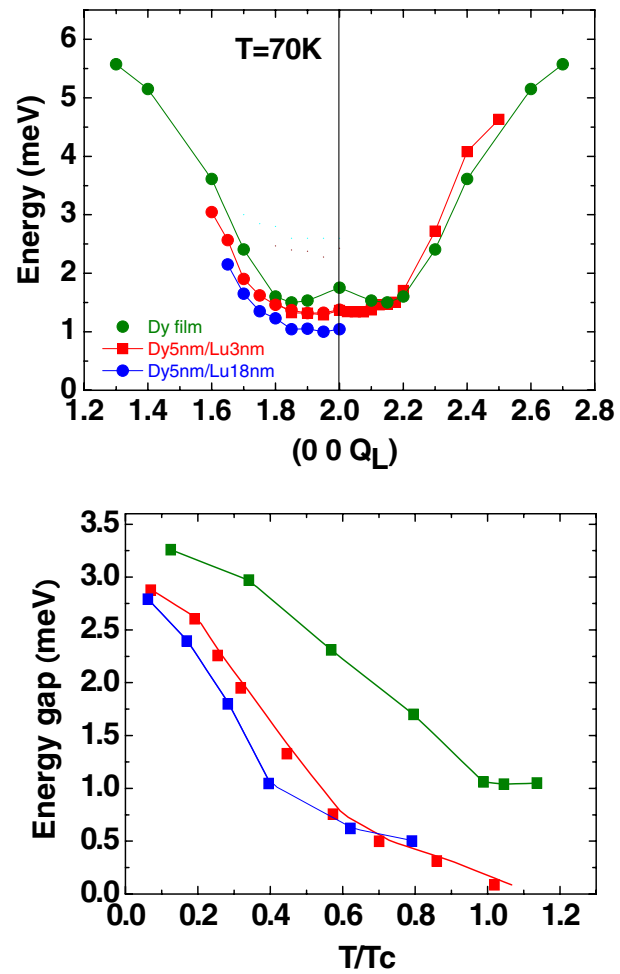

Figure 4. Top: dispersion curves measured at $70 \mathrm{~K}$ for the two Dy/Lu superlattices, compared to the Dy thick film. Bottom: energy gap versus reduced temperature measured for the two Dy/Lu superlattices, compared to the Dy thick film.

SL's dispersion curves exhibit rather similar shape with the presence of an energy gap, but two obvious differences with thick Dy film have to be underlined:

- The energy gap in SL's appears to be smaller and to present unusual temperature dependence with two regimes in temperature, as shown in Figure 4, bottom (note the reduced temperature $\mathrm{T} / \mathrm{T}_{\mathrm{c}}$ used as $\mathrm{x}$-scale). The gap is obviously smaller in SL's at the onset of ferromagnetism $\left(\mathrm{T} / \mathrm{T}_{\mathrm{c}}=1\right)$ and increases more slowly when $\mathrm{T}$ decreases down to bulk $\mathrm{T}_{\mathrm{c}}\left(\mathrm{T} / \mathrm{T}_{\mathrm{c}} \approx 0.55\right)$. Below bulk $\mathrm{T}_{\mathrm{c}}$, the increase in energy gap becomes steeper and more similar to bulk. This likely points out the specific nature of the "above bulk $\mathrm{T}_{\mathrm{c}}$ " ferromagnetic phase in SL's. Note that the gap is smaller in SL's, even a low temperature, and smaller in the case of thicker Lu spacer. This suggests a modification of anisotropy/magnetoelastic contributions that may depend on epitaxial strains. Such effects should now be included in the calculation to achieve a more accurate and quantitative description.

- There is an obvious "flattening" of SL's dispersion curves around (002). A close inspection reveals that minima on each side of (002) are still present in SL's but the difference between energies at those positions and at (002) is much smaller than in the Dy film. This would suggest that the difference in exchange energy contributions between modulated and collinear orders is reduced, i.e. the modulated order is less favored by exchange than in bulk Dy. Such feature is consistent with conclusions drawn from measurements of turn angle in Dy/Er bilayers [13]. It could be related to changes in the Dy Fermi surface and likely contributes to the increase of $\mathrm{T}_{c}$ in SL's.

Spin waves calculations for low dimension systems predict interesting features related to reduced dimensions and possible magnetic coupling, as it is shown in Figure 5. The calculations have been 


\section{Collection SFN}

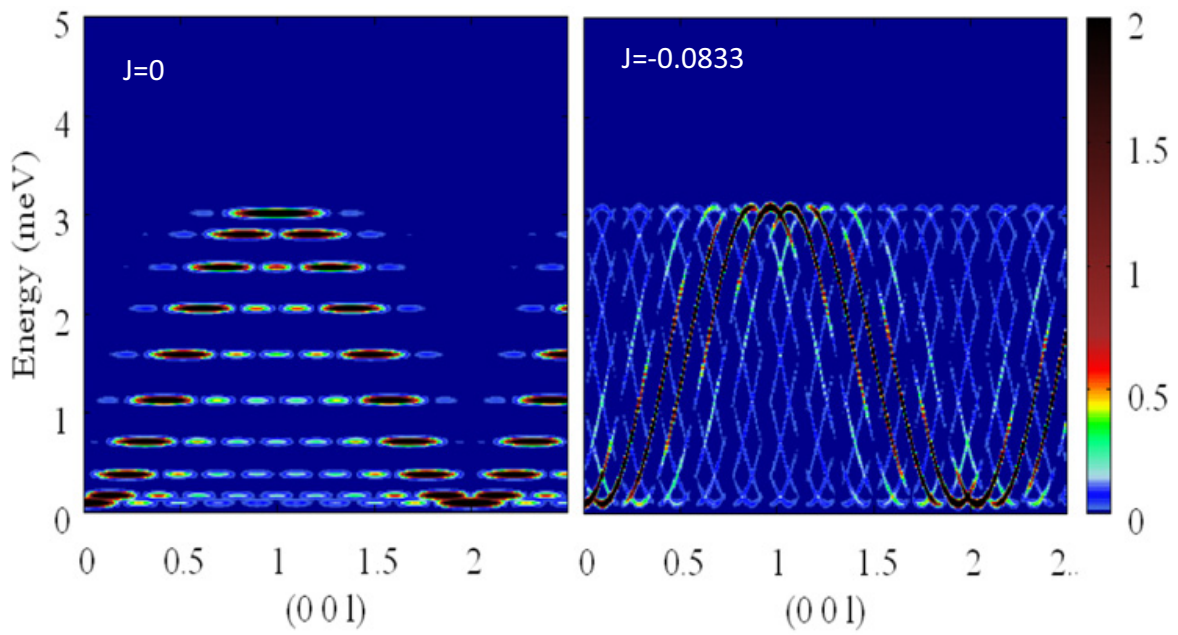

Figure 5. Dispersion curves calculated for a stacking of 10 atomic planes thick Dy layers in the ferromagnetic phase, in the cases where Dy layers are decoupled from each other (left) and ferromagnetically coupled (right).
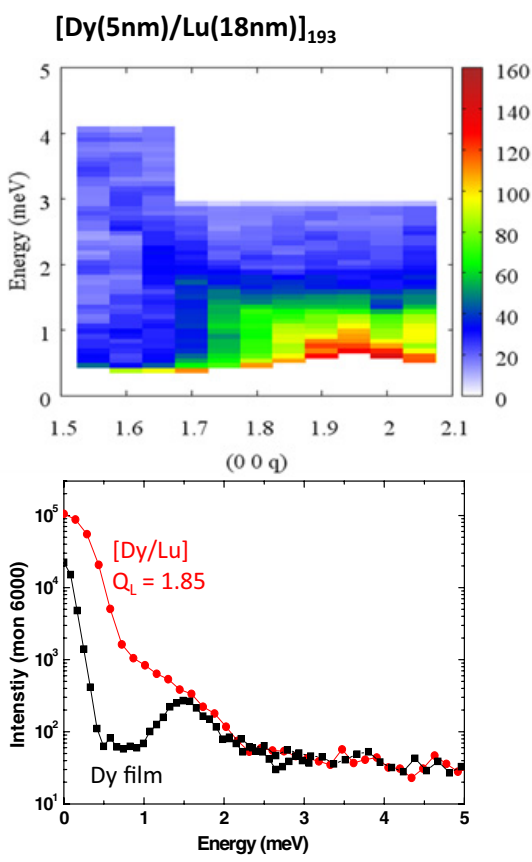

$[\operatorname{Dy}(5 \mathrm{~nm}) / \mathrm{Lu}(3 \mathrm{~nm})]_{597}$
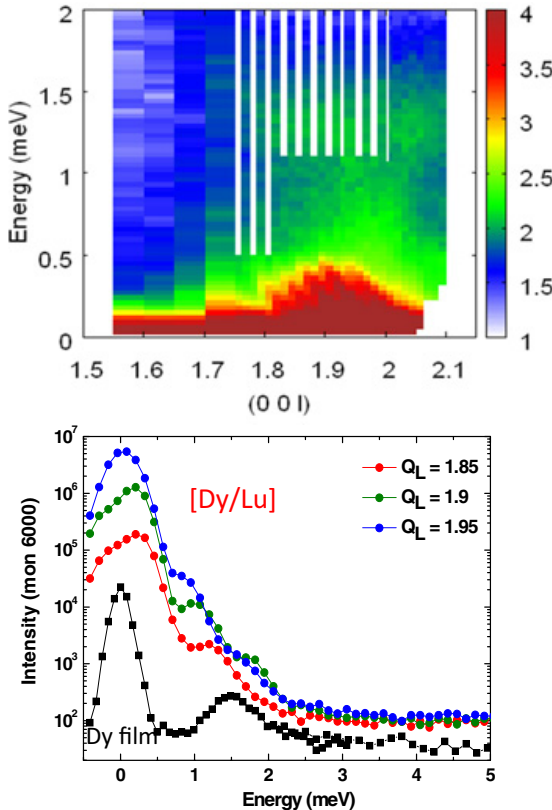

Figure 6. Inelastic Neutron Scattering results at $70 \mathrm{~K}$ from $[\mathrm{Dy}(5 \mathrm{~nm}) / \mathrm{Lu}(18 \mathrm{~nm})]_{193}$ (left) and $[\mathrm{Dy}(5 \mathrm{~nm}) /$ $\mathrm{Lu}(3 \mathrm{~nm})]_{597}$ (right) superlattices. In top row are $(\mathrm{E}, \mathrm{Q})$ intensity maps. In bottom row are E scans collected for different $\left(\begin{array}{lll}0 & 0 & Q_{L}\end{array}\right)$ positions in comparison to Dy thick film measured at $\left(\begin{array}{lll}0 & 0 & 2.15\end{array}\right)$.

performed for a stacking of 10 atomic planes thick Dy layers for two different coupling values: $\mathrm{J}=0$ and $\mathrm{J}=-0.0833$.

Reducing the Dy layers thickness to 10 atomic planes yields discontinuous, discrete and flat energy levels, the separation of which is related to individual layers thickness. The intensity distributed on these 
levels makes however that overall bulk signature remains. When coupling is activated between those thin Dy layers (Fig. 5, right), individual excitations become dispersive again and break into multiple branches. The splitting of Spin Waves branches is related to the bilayer thickness, and the intensity is still mainly distributed along the bulk dispersion curve.

Investigation of those specific features was an important but difficult task of the study. Figure 6 gathers the results obtained at $70 \mathrm{~K}$ for the two SL's with $\mathrm{kf}=1.3 \AA^{-1}$ in order to increase the energy resolution to $0.1 \mathrm{meV}$. The discretisation of energy levels expected in the SL with $18 \mathrm{~nm}$ thick Lu spacer layers remains obscure (Fig. 6, top left), although the shape of the energy scan measured for $\left(\begin{array}{lll}0 & 0 & 1.85\end{array}\right)$ (bottom left) is obviously different from Dy thick film. This may suggest some supplemental structures and possibly multiple energy levels, but a similar sample with larger total amount of Dy is definitively required. Results from the SL with thinner spacer layers and a larger amount of Dy are more convincing. The (energy, Q) map presented here (Fig. 6, top right) with a logarithm intensity scale exhibits several parallel and extended features that could be attributed to split SW branches. Energy scans recorded at different Q positions (bottom right) confirm the presence of multiple branches that disperse in Q, as expected from the BZ folding effect.

Those INS measurements on Dy-based superlattices first prove that such measurements can be undertaken for the investigation of spin waves and fundamental interactions in nanosystems. If the effect of reduced dimensions on the discretization of energy levels in SL still remains unclear from those experiments, the observation of split SW branches expected from magnetic coupling represents an interesting progress in the field. Dispersion curves also suggest a possible effect of epitaxial strains on exchange, anisotropy and magnetoelastic constant that definitely motivates further exploration and calculation. New exploration of spin dynamics of nanosystems can thus be reasonably undertaken in wide regions of the Brillouin zone. Higher flux neutron sources and still improved instruments with higher energy resolution will most likely enable the study of wider range of nanosystems where spin dynamics is an essential issue for technological applications.

\section{References}

[1] K. Dumesnil and S. Andrieu, "Molecular Beam Epitaxy: From research to mass production" chapter 20: "Epitaxial magnetic layers grown by MBE: model systems to study the physics in nanomagnetism and spintronic", ed. M. Henini, Elsevier (2012).

[2] J.M.D. Coey, "Magnetism and Magnetic Materials", Cambridge University Press (2009).

[3] N.H. Duc, "Giant Magnetostriction in Lanthanide-transition metal films thin films", in "Handbook on the Physics and Chemistry of rare earths", vol 32, K.A. Gschneider ed. (2001).

[4] C. Kaiser, A.F. Panchula, and S.S.P. Parkin, Phys. Rev. Lett. 95, 047202 (2005).

[5] A. Avisou, C. Dufour, K. Dumesnil, A. Rogalev, F. Wilhelm, E. Snoeck, J. Phys.: Condens. Matter 20, 265001 (2008).

[6] S. Qiao, A. Kimura, H. Adachi, K. Iori, K. Miyamoto, T. Xie, H. Namatame, M. Tanigushi, A. Tanaka, T. Muro, S. Imada, S. Suga., Phys. Rev. B 70, 134418 (2004).

[7] M.Da Silva, K. Dumesnil, C. Dufour, M. Hehn, D. Pierre, D. Lacour, F. Montaigne, G. Lengaigne, S. Robert, Appl. Phys. Lett. 98, 232504 (2011).

[8] G.L.J.A. Rikken J. Foelling, and P. Wyder, Phys. Rev. Lett. 87, 037203 (2001).

[9] J.W.A. Robinson, J.D.S. Witt, and M.G. Blamire, Science 329, 59 (2010).

[10] O. Wessely, B. Skubic, and Lars Nordström, Phys. Rev. Lett. 96, 256601 (2006).

[11] Fuxiang Li, T. Nattermann, and V.L Pokrovsky, Phys. Rev. Lett. 108, 107203 (2012).

[12] S.W. Chen, H. Guo, K.A. Seu, K. Dumesnil, S. Roy and S.K. Sinha, submitted for publication

[13] K. Dumesnil, C. Dufour, Ph. Mangin, G. Marchal, M. Hennion, Phys. Rev. B 54, 6407 (1996).

[14] B. Hennion, W. Szuszkiewicz, E. Dynowska, E. Janik, and T. Wojtowicz, Phys. Rev. B 66, 224426 (2002). 


\section{Collection SFN}

[15] A.T.D. Grunwald, A.R. Wildes, W. Schmidt, E.V. Tartakovskaya, J. Kwo, C. Majkrzak, R.C.C. Ward, and A. Schreyer, Phys. Rev. B. 82, 014426 (2010).

[16] M.K Wilkinson, W.C Koelher, E.O Wollan and J.W Cable, J. Appl. Phys. 32, 488 (1961).

[17] J.W Cable, E.O Wollan, W.C Koelher and M.K Wilkinson, Phys. Rev. 140, A1896 (1965).

[18] B. Coqblin, The electronic structure of the rare earth metals and alloys: The heavy rare earth (Academic, London, 1977).

[19] F.J. Darnel, Phys. Rev. 130, 1825 (1963).

[20] K. Dumesnil, C. Dufour, Ph. Mangin, G. Marchal and M. Hennion, Europhys. Lett. 31, 43 (1995).

[21] K. Dumesnil, C. Dufour, Ph. Mangin and G. Marchal, Phys. Rev. B 53, 11218 (1996).

[22] J.J. Rhyne and R.W. Erwin, "Magnetism in artificial metallic superlattices of rare earth metals" in Magnetic Materials vol. 8, ed. K.H.J. Bushow (1993).

[23] R.W. Erwin, J.J. Rhyne, M.B. Salamon, J. Borchers, S. Sinha, R. Du, J.E. Cunningham, and C.P. Flynn, Phys. Rev. B 35, 6808 (1987).

[24] R.S. Beach, J.A. Borchers, A. Matheny, R.W. Erwin, M.B. Salamon, B. Everitt, K. Pettit, J.J. Rhyne, and C.P. Flynn, Phys. Rev. Lett. 70, 3502 (1993).

[25] K. Dumesnil, C. Dufour, M. Vergnat, G. Marchal, Ph. Mangin, M. Hennion, W.T. Lee, H. Kaiser, J.J. Rhyne, Phys. Rev. B 49, 12274 (1994).

[26] K. Dumesnil, A. Stunault, Ph. Mangin, C. Vettier, D. Wermeille, N. Bernhoeft, S. Langridge, C. Dufour, and G. Marchal, Phys. Rev. B 58, 3172 (1998).

[27] M. Braden, B. Hennion, W. Reichard, G. Dhalenne and A. Revcolevschi, Physica B, 241-243, 531 (1998).

[28] M. Hennion et al, Phys. Rev. B 73104453 (2006), M. Hennion et al., Phys. Rev. Lett. 94057006 (2005).

[29] H. Béa, M. Bibes, F. Ott, B. Dupé, X.-H. Zhu, S. Petit et al,, Phys. Rev. Lett. 100, 017204 (2008).

[30] S. Petit, F. Moussa, M. Hennion, S. Pailhés, L. Pinsard-Gaudart, et al., Phys Rev Lett. 99, 266604 (2007).

[31] J.T. Haralden and R.S. Fishman, J. of Physics: Cond. Matter. 22, 186002 (2010).

[32] R.M. Nicklow, J. Appl. Phys. 42, 1672 (1971); R.M. Nicklow, N. Wakabayashi, M.K. Wilkinson, R.E. Reed, Phys. Rev. Lett. 26, 140 (1971).

[33] H.B Moller, J.C. Gylden Houmann and A.R. Mackintosh, J. of Appl. Physics 39, 807 (1968).

[34] M.S.S. Brooks, Phys. Rev. B 1, 2257 (1970). 